\title{
Optimizing Burr Hole Placement for Craniotomy: A Technical Note
}

\author{
Survendra Kumar Rajdeo Rai ${ }^{1} \quad$ Saswat Kumar Dandpat ${ }^{1} \quad$ Dikpal Jadhav $^{1} \quad$ Shashi Ranjan ${ }^{1}$ \\ Abhidha Shah $^{1}$ Atul H. Goel ${ }^{1}$
}

${ }^{1}$ Department of Neurosurgery, King Edward Memorial Hospital and Seth Gordhandas Medical College, Mumbai, Maharashtra, India

Address for correspondence Survendra Kumar Rajdeo Rai, DNB, Department of Neurosurgery, King Edward Memorial Hospital and Seth Gordhandas Medical College, Acharya Donde Marg, Parel, Mumbai 400 012, Maharashtra, India (e-mail: rskrrai@gmail.com).

\begin{abstract}
Keywords

- beveled edge

- bone flap

- craniotomy

- optimal burr hole

Objective Usually, burr holes are placed along the line of a craniotomy. We describe a novel technique of burr hole placement to obtain smooth and beveled bony margin without any troughs and crests. Dural separation is obtained by minimizing the number of burr holes required.

Methods Fifty craniotomies of diameter ranging from 3.5 to $11.5 \mathrm{~cm}$ were accomplished by placing burr hole in the center of bone flap rather than along the craniotomy line permitting 360 degrees of dura separation dependent on the length of dura separator. Craniotomy $<9 \mathrm{~cm}$ in diameter was performed by placing a single burr hole and a larger size craniotomy was performed with two burr holes. Parasagittal craniotomy was performed by placing burr hole not $>2.5 \mathrm{~cm}$ away from expected craniotomy site, namely superior sagittal sinus area enabling separation of adhered dura and venous sinuses. The bone cutter was used in a particular fashion to create smooth margin and beveled edges.

Results Craniotomy $<9 \mathrm{~cm}$ in diameter was possible with single burr hole in 34 cases. Craniotomy larger than $9 \mathrm{~cm}$ in size was performed in 16 cases with double burr hole by strategically placing burr in the center of the desired bone flap. The craniotomy was achieved in all cases without damaging dura and venous structures.

Conclusions An optimally placed single burr hole is sufficient for small to moderately large size craniotomy. Larger size craniotomy is possible with minimum numbers of burr holes. This achieves good cosmesis and avoids sinking of the bone flap.
\end{abstract}

\section{Introduction}

A craniotomy is a sine qua non in all open approaches to deal with various pathology of the brain. As neurosurgery has evolved, we have advanced from techniques such as trephination and manual Hudson brace to electrical/pneumatic high-powered drills which have made opening of the skull easy.

Performing burr hole is the initial step for accomplishing the craniotomy. Site of burr hole placement is of utmost importance for separating the underlying dura as well as maximizing the craniotomy. Conventionally, burr holes are placed over the corners of the desired craniotomy which are along the craniotomy lines. Sometimes, it is also necessary to add additional burr holes if dura is expected to be densely adherent as in case of elderly or when the craniotomy is of large size.

Bone flap developed by routine technique has irregular bony margins with troughs created especially at the sites where burr hole was made. Usually, single burr hole or a smaller number of burr holes are utilized for craniotomy in relatively young patients where dura is free from the underlying bone. In older patients where the dura is densely adherent, there is a tendency to use more burr holes than routinely required for the separation of the dura. The defect is more pronounced if multiple burr holes are made for the craniotomy. 
We describe a technique of burr hole placement which will create a smooth, beveled margin with better fitting of bone flap to the craniotomy defect and better cosmesis.

\section{Methods}

From January 2018 to September 2018, 50 patients with brain tumors located in different areas were treated using the described technique of burr hole placement ( $\mathbf{- T a b l e} \mathbf{1}$ ). All the procedures were performed at the Department of Neurosurgery, King Edward Memorial Hospital and Seth Gordhandas Medical College. Various pathologies treated included 24 cases of glioma, 23 cases of meningioma, and 3 cases of the colloid cyst. There were 9 frontal craniotomies, 7 parietal, 5 temporal, 11 frontoparietal, 4 parieto-occipital, and 14 parasagittal craniotomies (-Table $\mathbf{1}$ ). There were 29 males and 21 females. Age of the patients ranged from 4 to 79 years (mean: 44.7 years) (-Table 2). Patients requiring craniotomy for lateral basal subfrontal craniotomy were not included in the study as in these situations burr hole is placed underneath the temporalis muscle. Furthermore, cases requiring reoperation were excluded from this study.

Using this technique, the placement of the burr hole site was modified so as to reduce the number of burr holes. Smooth bony margins along the craniotomy lines were achieved using this technique ( $\boldsymbol{- F i g s .} \mathbf{1}$ and $\mathbf{2}$ ). Rather than placing the burr hole along the corners of the square/rectangular bone flap, we placed the burr hole in the center of the bone flap. Then, the dura was separated all-around the burr hole site in a 360-degree fashion. It was possible to separate approximately 2.5 to $4 \mathrm{~cm}$ of adjoining dura from the burr hole margin. It was possible to perform around $<9 \mathrm{~cm}$ diameter size craniotomy with just one burr hole placement (-Figs. 1 and $\mathbf{2}$ ). The extent of dura separation depends on the size and type of the dural separators. Depending on the size of craniotomy, desired number of burr holes can be placed away from the actual site of the craniotomy such that the underlying dura is separated from the skull bone (-Fig. 2). With the aid of an electric or pneumatic drill, the craniotomy was fashioned (-Figs. 1 and $\mathbf{2}$ ). The burr holes were filled with bone dust after completion of surgery ( - Figs. 3A, B). As there are no burr holes along the craniotomy line, bony margins are smooth without any troughs and crests. There is less bone loss as compared with the conventional technique involving multiple burr holes for the same size craniotomy.
Table 2 Age and sex distribution of the patients in the series

\begin{tabular}{|l|l|l|}
\hline \multirow{2}{*}{ Age $(y)$} & \multicolumn{2}{|c|}{ Sex } \\
\cline { 2 - 3 } & Male & Female \\
\hline $1-20$ & 1 & 2 \\
\hline $21-40$ & 9 & 6 \\
\hline $41-60$ & 12 & 9 \\
\hline $61-80$ & 7 & 4 \\
\hline
\end{tabular}

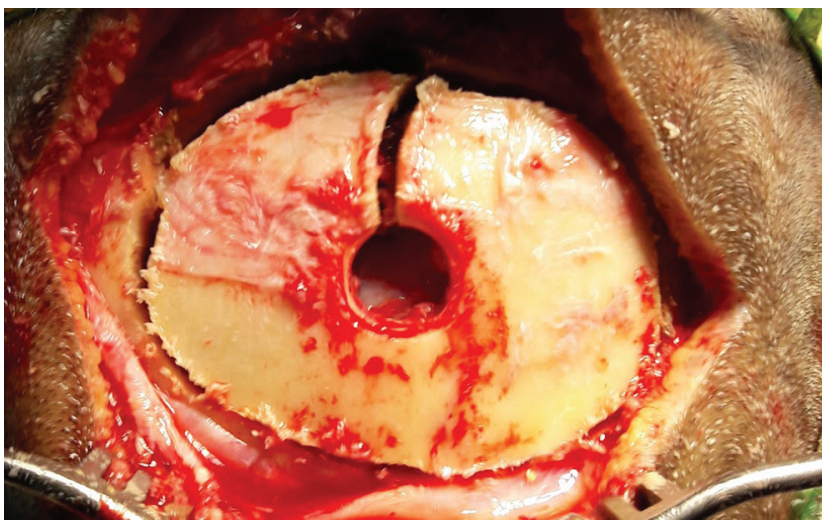

Fig. 1 Single burr hole is seen in the center of the bone flap. The bone cutter is used in a fashion to obtain smooth margin.

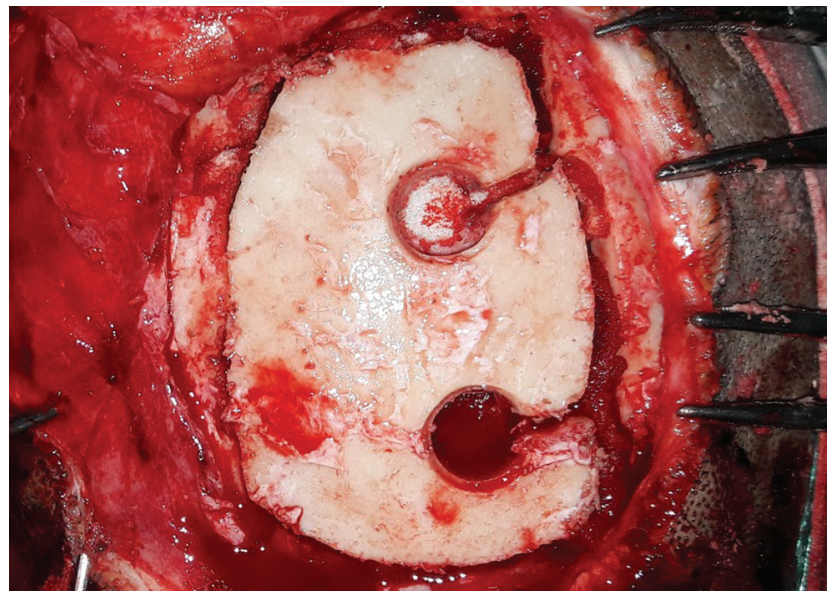

Fig. 2 Parasagittal craniotomy with two burr holes approximately $2.5 \mathrm{~cm}$ away from the sinus are used to separate dura over the sinus effectively to obtain smooth margin bone. Burr hole directly over the sinus is avoided.

Table 1 Location and number of craniotomy performed for various pathology using single or double burr hole technique

\begin{tabular}{|l|l|l|l|l|l|}
\hline \multirow{2}{*}{ Location of craniotomy } & \multicolumn{3}{|c|}{ Pathology } & \multicolumn{2}{c|}{ Number of burr holes } \\
\cline { 2 - 6 } & Glioma & Meningioma & Colloid cyst & Single burr hole & Double burr hole \\
\hline Frontal & 5 & 4 & & 9 & \\
\hline Parietal & 4 & 3 & & 7 & \\
\hline Temporal & 2 & 3 & & 5 & 5 \\
\hline Frontoparietal & 5 & 6 & & 6 & \\
\hline Parieto-occipital & 2 & 2 & & 4 & 11 \\
\hline Parasagittal & 6 & 5 & 3 & 3 & \\
\hline
\end{tabular}




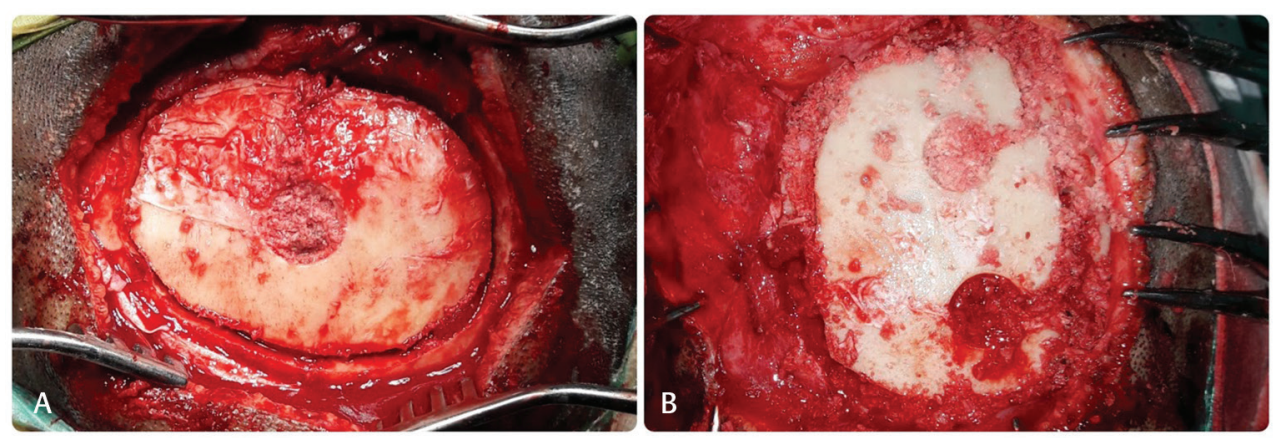

Fig. 3 Burr hole defect and craniotomy line is filled with bone dust as seen in case of craniotomy with single burr hole (A) and parasagittal craniotomy with two burr holes (B).

\section{Results}

The sizes of the craniotomy ranged from 3.5 to $11.5 \mathrm{~cm}$ (mean: $7.1 \mathrm{~cm}$ ). It was possible to achieve $<9 \mathrm{~cm}$ diameter size craniotomy with just single burr hole placed at the center of the desired bone flap, while it was possible to achieve craniotomy of size $>9 \mathrm{~cm}$ in diameter or more with the placement of two burr holes. Cases requiring larger parasagittal craniotomy $>9 \mathrm{~cm}$ in diameter especially in cases of meningiomas and gliomas were achieved with double burr holes without damaging the underlying dura and venous sinuses. The craniotomy was performed with single burr hole in 34 cases and double burr holes in 16 cases (-Table 1). It was possible to achieve the desired craniotomy in all the cases preserving the underlying dura and venous sinuses. Cosmetic results of all patients were excellent without any sinking of the bone flap.

\section{Discussion}

Craniotomy by conventional technique involves multiple burr holes along the craniotomy line. Although multiple burr holes help in dura separation from the overlying bone flap, having burr holes along the craniotomy line gives irregular and sharp edges. If the bone flap is not approximated well either with the suture or screws and plates, bony irregular edges tend to protrude out from the scalp or sometimes even hurt the overlying skin or get exposed if eroded, particularly in cases where the bone flap is under severe pressure due to any underlying mass/growth.

The dura underlying the bone can be separated all-round the burr hole site. However, burr hole located at the corners of the craniotomy utilizes only one-fourth (90 degrees) dura separating capability and burr holes placed along the line of craniotomy in between two corners utilizes only half (180 degrees) of the potentiality to separate the dura. The placement of burr hole in the center of the desired craniotomy flap permits separation of the dura from the overlying bone to the full extent (360 degrees) and is technically equivalent to that achieved by placing four burr holes at the four corners of the craniotomy. Depending on the size, site, and location of the bone flap, burr holes are always placed meticulously away from the actual craniotomy line such that dura separating potential of the burr hole is utilized to the full extent and the number of burr holes is minimized to achieve the same goal. We found that even for large bone flaps, number of burr holes required for the craniotomy is less than that required by routine technique. In craniotomy along the superior sagittal sinus, burr holes are placed not $>2.5 \mathrm{~cm}$ away from the actual craniotomy line lying over the sinuses such that venous sinuses can be easily separated. In cases of elderly patients with tendency of dense dura adhesion, burr holes were made approximately $1 \mathrm{~cm}$ away from the actual craniotomy site. A narrow bony defect along all the sides of the craniotomy ensures good approximation with the surrounding bone either by suturing the bone flap or anchoring it with the screw and plates. Furthermore, there is potential for earlier fusion with the surrounding bone with better cosmesis. Burr hole being in the center of the bone flap can easily be filled up with bone dust. Even if the bone dust is not available for filling this defect, these burr holes do not add to the cosmetic deformity as these are small in size and are surrounded by significant intact skull bone. During craniotomy, care should be taken to ensure that the craniotomy was done in slanting/beveled fashion so that the bone flap fits well to the craniotomy defect and prevents sinking inside the cranial cavity. ${ }^{1,2}$

\section{Conclusion}

Optimally placed single burr hole is sufficient for small to moderately large size craniotomy, while larger size craniotomy is possible with minimum numbers of burr holes and with better cosmesis. Slanting/beveling of the bone flap avoids its sinking postoperatively.

\section{Funding}

None.

\section{Conflict of Interest}

None declared.

\section{Acknowledgments}

The authors would like to thank Mr. Karun Keshav Bandekar, operation theater staff, for his help in taking intraoperative photographs and Dr. Cristina Mancarella, Neurosurgeon, IRCCS NEUROMED-Mediterranean Neurological Institute, Italy, for her constant encouragement and support. 


\section{References}

1 DiMeco F, Li KW, Mendola C, Cantú G, Solero CL. Craniotomies without burr holes using an oscillating saw. Acta Neurochir (Wien) 2004;146(9):995-1001, discussion 1001

2 Van Dijk JM, Thomeer RT. Use of the Gigli saw in performing a mid-frontobasal or pterional craniotomy. Br J Neurosurg 1997;11(6):558-559 